\title{
Pseudoscalar meson physics with four dynamical quarks
}

\section{A. Bazavov, ${ }^{a}$ C. Bernard, ${ }^{b}$ C. Bouchard,${ }^{c}$ C. DeTar, ${ }^{d}$ D. Du, ${ }^{e}$ A.X. El-Khadra, ${ }^{e}$} J. Foley, ${ }^{d}$ E.D. Freeland, ${ }^{f}$ E. Gamiz,${ }^{g}$ Steven Gottlieb, ${ }^{h}$ U.M. Heller, ${ }^{i}$ J.E. Hetrick, ${ }^{j}$ J. Kim, ${ }^{k}$ A.S. Kronfeld, ${ }^{l}$ J. Laiho, ${ }^{m}$ L. Levkova, ${ }^{d}$ M. Lightman, ${ }^{b}$ P.B. Mackenzie, ${ }^{l}$ E.T. Neil, ${ }^{l}$ M. Oktay, ${ }^{d}$ J.N. Simone, ${ }^{l}$ R.L. Sugar ${ }^{n}$ D. Toussaint ${ }^{* k}$ R.S. Van de Water, ${ }^{a \dagger}$ and R. Zhou ${ }^{h}$ [Fermilab Lattice and MILC Collaborations]

${ }^{a}$ Department of Physics, Brookhaven National Laboratory, Upton, NY 11973, USA

${ }^{b}$ Department of Physics, Washington University, St. Louis, MO 63130, USA

${ }^{c}$ Department of Physics, The Ohio State University, Columbus, OH 43210, USA

${ }^{d}$ Physics Department, University of Utah, Salt Lake City, UT 84112, USA

e Physics Department, University of Illinois, Urbana, IL 61801, USA

${ }^{f}$ Department of Physics, Benedictine University, Lisle, IL 60532, USA

${ }^{g}$ CAFPE and Departamento de Fisica Te orica y del Cosmos, Universidad de Granada, Granda, Spain

${ }^{h}$ Department of Physics, Indiana University, Bloomington, IN 47405, USA

${ }^{i}$ American Physical Society, One Research Road, Ridge, NY 11961, USA

${ }^{j}$ Physics Department, University of the Pacific, Stockton, CA 95211, USA

${ }^{k}$ Physics Department, University of Arizona, Tucson, AZ 85721, USA

${ }^{l}$ Fermi National Accelerator Laboratory, Batavia, IL 60510, USA

${ }^{m}$ SUPA, School of Physics and Astronomy, University of Glasgow, Glasgow G12 8QQ, UK

${ }^{n}$ Department of Physics, University of California, Santa Barbara, CA 93106, USA

E-mail: dougephysics.arizona.edu,

\begin{abstract}
We present preliminary results for light, strange and charmed pseudoscalar meson physics from simulations using four flavors of dynamical quarks with the highly improved staggered quark (HISQ) action. These simulations include lattice spacings ranging from 0.15 to $0.06 \mathrm{fm}$, and seaquark masses both above and at their physical value. The major results are charm meson decay constants $f_{D}, f_{D_{s}}$ and $f_{D_{s}} / f_{D}$ and ratios of quark masses. This talk will focus on our procedures for finding the decay constants on each ensemble, the continuum extrapolation, and estimates of systematic error.
\end{abstract}

The 30th International Symposium on Lattice Field Theory - Lattice 2012

June 24-29, 2012

Cairns, Australia

\footnotetext{
* Speaker.

$\dagger$ Present address: Fermi National Accelerator Laboratory, Batavia, IL, USA
} 


\section{Introduction}

Lattice calculations of pseudoscalar meson decay constants, when combined with measured nonleptonic decay rates, can be used to extract CKM matrix elements and test the Standard Model. The lattice calculation includes a determination of the quark masses - fundamental parameters of the Standard Model. Here we present preliminary results for the charm meson decay constants $f_{D}$ and $f_{D_{s}}$, and the ratios of quark masses $m_{c} / m_{s}$ and $m_{u} / m_{d}$.

These calculations use lattices generated with the one-loop and tadpole improved Symanzik gauge action [1] and the HISQ fermion action [2]. We include four flavors of dynamical sea quarks: degenerate up and down, strange, and charm. Iterated smearing in the HISQ action reduces taste violations by a factor of three relative to the asqtad action. An improved charm-quark dispersion relation allows us to treat it the same way as the lighter quarks. Our lattice ensembles include ensembles with approximately physical light-quark masses, with volumes as large as $5.6^{3} \mathrm{fm}^{3}$. For more details, see Refs $[3,4,5]$. The parameters of the ensembles used in this calculation are tabulated in J. Kim's talk [6]. In the first stage of analysis we fit pseudoscalar meson two-point correlators to extract masses and amplitudes from random wall operators for a set of valence-quark masses. (See Ref. [6].) In the second stage of the analysis, we interpolate or extrapolate in valencequark masses to find the tuned valence masses on each ensemble, and the decay constants evaluated at these valence masses, but at the sea-quark masses and lattice spacing of the individual ensemble. Then we combine the results of the different ensembles to make a continuum extrapolation and corrections for mistuned sea-quark masses.

We use the pion decay constant $f_{\pi}$ to set the scale. Since this is determined from the same set of correlators as the charm meson decay constants, the analysis is self-contained in the sense that we do not need an intermediate quantity such as $r_{0}$ or $r_{1}$ found in a separate analysis. This reduces the error from determining the lattice spacing, and simplifies the error analysis. Ensembles with physical light-quark masses dominate the analysis and allow a simple interpolation to adjust for light-quark mass corrections. However, it is likely that use of staggered chiral perturbation theory will lead to better controlled fits, and J. Komijani's talk describes progress in this direction [7].

\section{Decay constants on each ensemble}

In the first stage of the analysis [6] we determine the masses and amplitudes for the two-point pseudoscalar correlators for a set of valence quark masses on each ensemble. These masses include 0.9 and 1.0 times the sea charm quark mass, 0.8 and 1.0 times the sea strange-quark mass, and a range of lighter masses. Since the sea-quark masses were estimated before the production runs began, they are inevitably slightly mistuned. In the second stage of the analysis we determine tuned quark masses for each ensemble, and evaluate the decay constants at these valence-quark masses. Our primary analysis uses $f_{\pi}$ to fix the lattice spacing, and proceeds as follows. To estimate statistical errors, this whole procedure is done inside a jackknife resampling.

1. Interpolate or extrapolate in the light valence-quark mass $m_{l}$ to the point where $\frac{M_{\pi}}{f_{\pi}}$ has its physical ${ }^{1}$ value. This fixes $a m_{l}$ and the lattice spacing $a$.

2. Interpolate or extrapolate in valence strange-quark mass $a m_{s}$ to where $2 M_{K}^{2}-M_{\pi}^{2}$ has its physical value. This fixes $m_{s}$.

\footnotetext{
${ }^{1}$ adjusted for E\&M and finite size — see later
} 


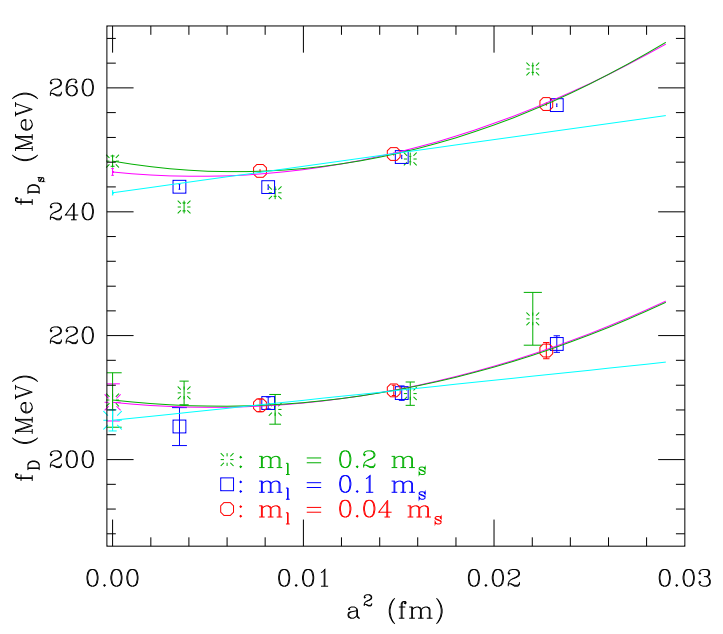

Figure 1: $f_{D}$ and $f_{D_{s}}$ on the different ensembles. Fits that are quadratic and linear in $a^{2}$, or use only physical quark mass ensembles are superimposed on the data. More details are given in the text.

3. Interpolate or extrapolate to the charm valence mass where $M_{D_{s}}$ is correct. This fixes $m_{c}$.

4. Find $m_{d}-m_{u}$ from E\&M adjusted $K^{0}-K^{+}$mass difference [8]

5. Find, by interpolation or extrapolation, $f_{K}$ at the adjusted up-quark mass.

6. Find $f_{D}$ and $M_{D}$ (a check) at the adjusted down and charm masses.

7. Find $f_{D_{s}}$ at the adjusted strange and charm masses.

8. Find $M_{\eta_{c}}$ (check) at the adjusted charm-quark mass.

We have also used a tuning procedure where we replaced $f_{\pi}$ and $M_{\pi}$ by the decay constant, $f_{p 4 s}$, and mass, $M_{p 4 s}$, of a fictitious meson with degenerate valence quark masses at 0.4 times the strange-quark mass, using the values for $f_{p 4 s}$ and $M_{p 4 s}$ in $\mathrm{MeV}$ determined from three flavor asqtad data. Finally, we varied the procedure by first fixing the lattice spacing from the static-quark potential, and then tuning the valence quark masses as described above.

In Eq. 2.1 we list results from the above procedure for the most influential ensemble ( $a \approx 0.09$ $\mathrm{fm}$ with physical masses). These results are adjusted for finite size and and some electromagnetic effects, but are not adjusted for mistuned sea-quark masses or extrapolated to the continuum limit. The errors here are statistical only but, as discussed above, errors from scale setting and valencequark mass tuning are included in the statistical error here. The $D^{+}, D^{0}$ and $\eta_{c}$ masses are not used in the tuning, so they can be compared to their experimental values, shown in parentheses.

$$
\begin{array}{llll}
a=0.08792(10) \mathrm{fm} & a m_{l}=0.001333(5) & a m_{s}=0.03648(11) & a m_{c}=0.4323(7) \\
m_{u} / m_{d}=0.480(6) & m_{s} / m_{l}=27.36(3) & m_{c} / m_{s}=11.851(17) & \\
f_{K}=155.12(22) \mathrm{MeV} & & \\
M_{D_{0}}=1867.8(1.5) \mathrm{MeV}(\operatorname{cf} 1864.8) & M_{D^{+}}=1870.2(1.2) \mathrm{MeV}(\operatorname{cf} 1869.6) \\
M_{\eta_{c}}=2980.2(3) \mathrm{MeV}(\operatorname{cf} 2980.3(1.2)) & & \\
f_{D}=208.75(1.03) \mathrm{MeV} \quad f_{D_{s}}=246.60(20) \mathrm{MeV} & f_{D_{s}} / f_{D}=1.181(6)
\end{array}
$$

\section{Continuum extrapolation}

The results for $f_{D}$ and $f_{D_{s}}$ on each ensemble are shown in Fig. 1. In this figure the physical quark mass ensembles, labelled as " $m_{l}=0.04 m_{s}$ ", have the smallest statistical errors. This is 

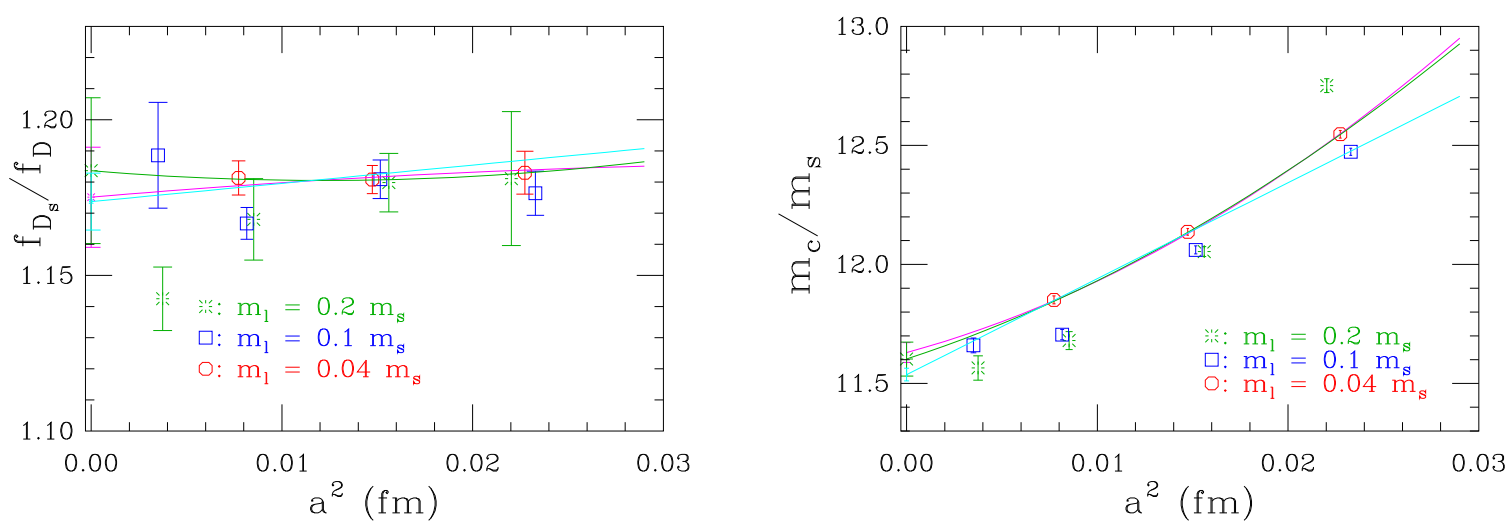

Figure 2: The ratios $f_{D_{s}} / f_{D}$ (left panel) and $m_{c} / m_{s}$ (right panel) on the different ensembles. The superimposed fit functions are the same forms as in Fig. 1.

due to their larger physical volumes, where the random wall and Coulomb wall sources, together with the average over spatial position of the sink operator, result in better statistics. Errors are large on some of the $m_{l}=0.2 m_{s}$ ensembles because the lightest valence-quark mass used on these ensembles was $0.1 m_{s}$, so a significant extrapolation in valence light-quark mass was necessary. When these points are fit to functions of lattice spacing and sea-quark mass, the physical quark mass points dominate the fit. Indeed, we could get reasonable results by simply using the physical quark mass ensembles. In practice, we use the physical quark mass and the $m_{l}=0.1 m_{s}$ ensembles, where the $m_{l}=0.1 m_{s}$ ensembles allow correction for mistuning of the light sea-quark masses in the physical quark mass ensembles and, because of the $m_{l}=0.1 m_{s}$ ensemble at lattice spacing $a \approx 0.06 \mathrm{fm}$, help to determine the dependence on the lattice spacing.

Figure 1 shows three different fits to this data. The magenta line uses all four of the lattice spacings, and is quadratic in $a^{2}$ and linear in light sea-quark mass. The straight cyan line in Fig. 1 omits the $a=0.15 \mathrm{fm}$ points, and is linear in both $a^{2}$ and $m_{l, \text { sea }}$. The green line is a quadratic fit using only the physical quark mass points, with small adjustments to correct for sea-quark mass mistuning. The symbols at $a=0$ are the continuum limits of these fits, showing the statistical error. The size of these symbols is proportional to the P-value of the fit, with the symbol size in the graph legend corresponding to 50\%. Since we expect both order $a^{2}$ and $a^{4}$ corrections to the data, and since some other accurately determined quantities such as the mass of the $\eta_{c}$ clearly need $a^{4}$ terms to fit the results, we take the quadratic fit results as our central value, with the larger of the difference between the quadratic and linear fits or the difference between the quadratic and physical-quark-mass-only fits as an estimate of the systematic error coming from our choice of fit form.

Figure 2 shows, with the same notation, the ratios $f_{D_{s}} / f_{D}$ and $m_{c} / m_{s}$ on each ensemble, together with fits to the same functional forms and ranges of data as in Fig. 1.

\section{Systematic errors}

Statistical errors from the jackknife analysis incorporate errors in lattice spacing determination and valence-quark mass tuning, since this tuning is redone for each jackknife sample. The following additional systematic errors are included in our error budget. 

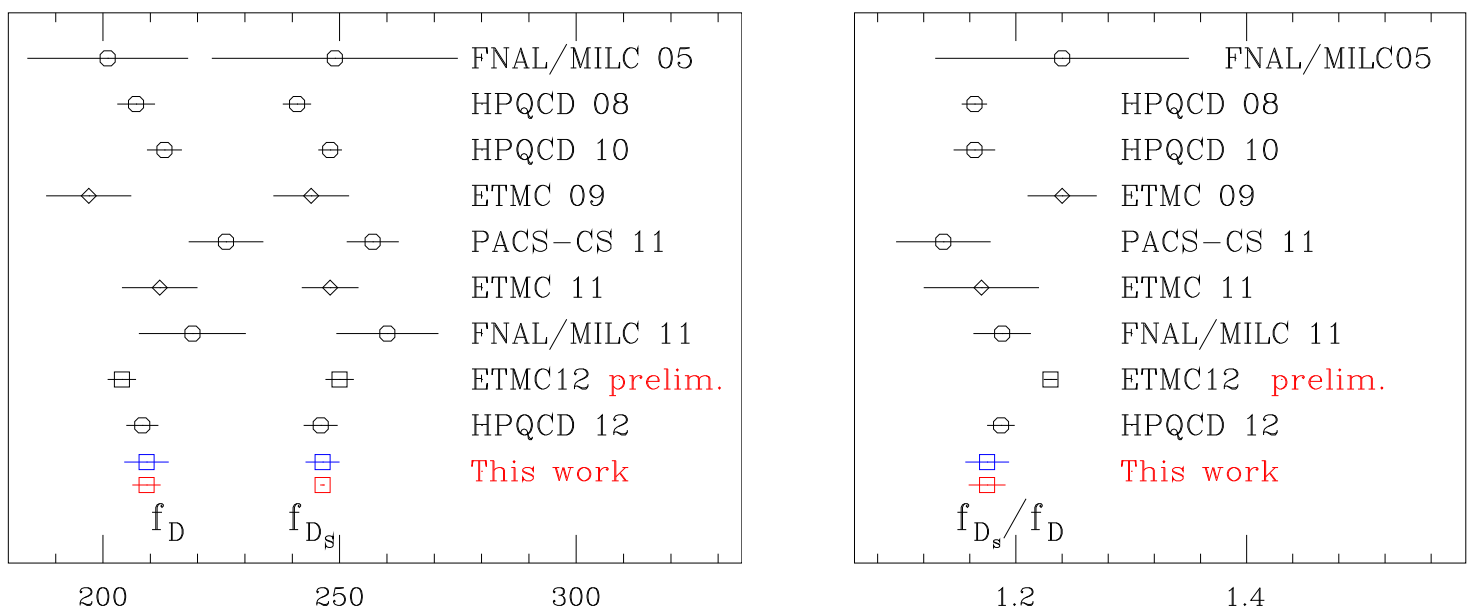

Figure 3: Comparison of lattice results for $f_{D}$ and $f_{D_{s}}$ (left panel) and for $f_{D_{s}} / f_{D}$ (right panel). Results are from Ref. [10] and this work. Diamonds represent results with 2, octagons with $2+1$, and squares with $2+1+1$ dynamical flavors. For this work the red error bars (lower) are statistical only, while the blue error bars (higher) include systematic error estimates.

Effects of excited states in the two-point correlators were estimated by varying the time range in the fits and the priors for excited state masses with reasonable ranges [6].

Effects of finite spatial size were tested by running otherwise identical ensembles with three different spatial sizes. This was done at $a \approx 0.12 \mathrm{fm}$ with $m_{l} / m_{s}=1 / 10$. The results, summarized in Fig. 4, show that the significant effects are in the light pseudoscalar sector, on $M_{\pi}, f_{\pi}$ and $f_{K}$, and that chiral perturbation theory is a reasonable guide. From these results, we estimated the effects on $M_{\pi}, f_{\pi}$ and $f_{K}$ at the physical light-quark mass in a $5.5 \mathrm{fm}$ box. (Since our fits are evaluated at this mass, it doesn't matter that the ensembles with other sea-quark masses would have different finite-size effects.) Then, in the analysis procedure described in section 2, we used the values of $M_{\pi}, f_{\pi}$ and $f_{K}$ adjusted to this size box. (That is, we compute $\frac{f_{K}}{f_{\pi}(5.5 \mathrm{fm})}$ etc.) Afterwards, we use these same factors to correct the computed $f_{K}$ back to its infinite volume value. We use the values with the finite-size adjustments as our central values, and take one half of the shifts when these adjustments are omitted as a remaining systematic error.

The effects of electromagnetic interactions and isospin breaking $\left(m_{u} \neq m_{d}\right)$ are considered together. We tune using the $\pi^{0}$ mass, where electromagnetic and isospin breaking effects are expected to be small. Then we use a separate calculation of electromagnetic effects on the kaon mass [8], whose main result is parameterized in terms of a violation of Dashen's theorem

$$
M_{K^{+}, a d j}^{2}=M_{K^{+}}^{2}-\left(1+\Delta_{E M}\right)\left(M_{\pi^{+}}^{2}-M_{\pi^{0}}^{2}\right)
$$

Here $M_{K^{+}, a d j}$ is the $K^{+}$mass adjusted to remove the effects of electromagnetism, and the result of Ref. [8] is $\Delta_{E M}=0.65(26)$. Then, in tuning the strange-quark mass from $2 M_{K}^{2}-M_{\pi}^{2}$, we use the average squared kaon mass $M_{K}^{2}=\left(M_{K^{+}, a d j}^{2}+M_{K^{0}}^{2}\right) / 2$. We also use this result combined with our meson mass measurements to determine the up-down quark mass difference,

$$
a^{2}\left(M_{K^{0}}^{2}-M_{K^{+}, a d j}^{2}\right)=a\left(m_{d}-m_{u}\right) \frac{\partial\left(a M_{K}\right)^{2}}{\partial a m_{l}},
$$



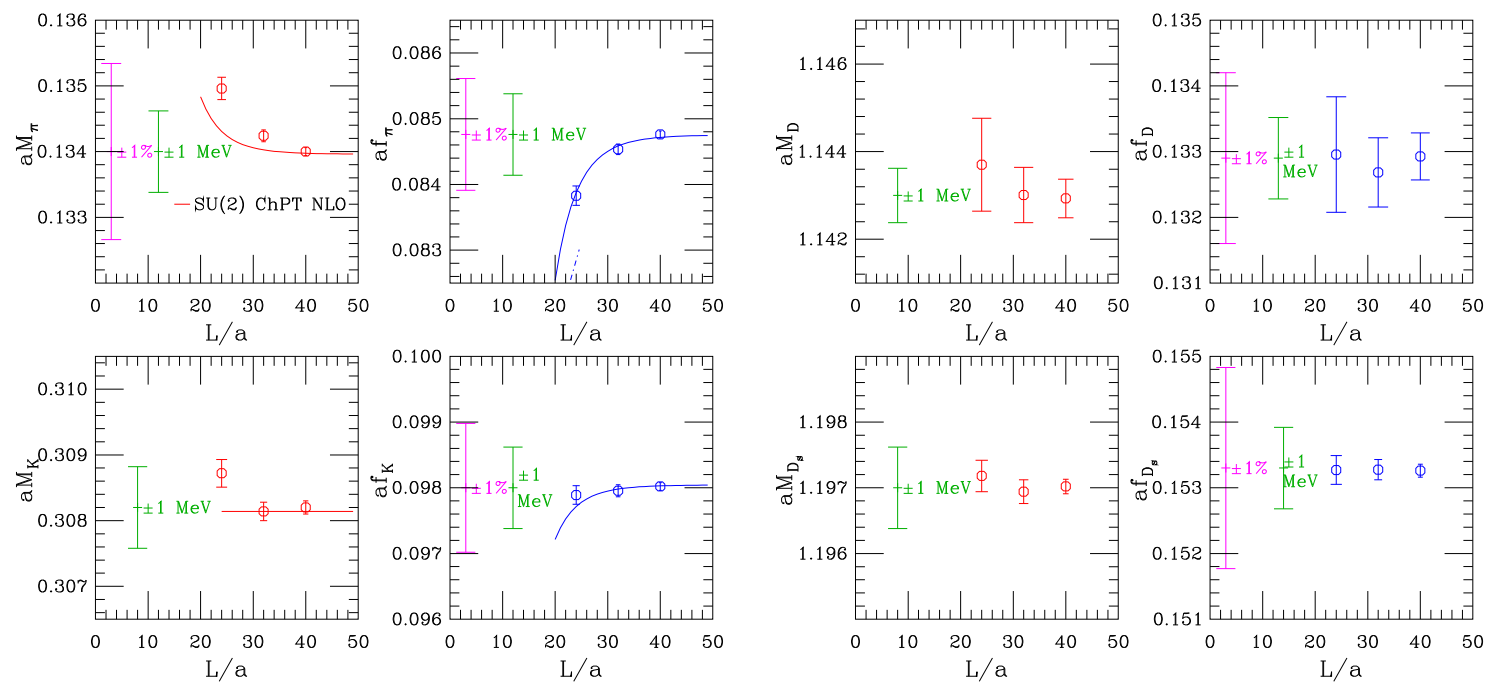

Figure 4: Spatial size effects on $M_{\pi}, M_{K}, f_{\pi}$ and $f_{K}$ (left side), and on $M_{D}, M_{D_{s}}, f_{D}$ and $f_{D_{s}}$ (right side). Solid lines are the one loop chiral perturbation theory forms. To show the magnitude of the effects, green error bars show an arbitrary value $\pm 1 \mathrm{MeV}$, and magenta error bars $\pm 1 \%$.

\begin{tabular}{|l|l|l|l|l|l|}
\hline Source & $f_{D}$ & $f_{D_{s}}$ & $f_{D_{s}} / f_{D}$ & $m_{c} / m_{s}$ & $m_{u} / m_{d}$ \\
\hline Stat. & $3.0 \mathrm{MeV}$ & $0.5 \mathrm{MeV}$ & 0.016 & 0.041 & 0.009 \\
Excited & $0.5 \mathrm{MeV}$ & $0.5 \mathrm{MeV}$ & 0.004 & 0.005 & 0.003 \\
Volume & $0.3 \mathrm{MeV}$ & $0.2 \mathrm{MeV}$ & 0.0004 & 0.008 & 0.0005 \\
E\&M & $0.04 \mathrm{MeV}$ & $0.12 \mathrm{MeV}$ & 0.001 & 0.001 & 0.021 \\
Scale set. & $2.0 \mathrm{MeV}$ & $1.3 \mathrm{MeV}$ & 0.003 & 0.02 & 0.023 \\
$a^{2}$ fit form & $2.9 \mathrm{MeV}$ & $3.3 \mathrm{MeV}$ & 0.010 & 0.09 & 0.011 \\
\hline
\end{tabular}

Table 1: Error budget: The statistical error includes error from valence-quark mass tuning and from a continuum extrapolation with fixed fit form. Estimation of the systematic error is discussed in the text.

where $\frac{\partial M_{K}^{2}}{\partial m_{l}}$ is obtained from the difference in $M^{2}$ between the two lightest valence-quark masses, with the strange mass set at the sea strange mass. We estimate the remaining systematic errors from electromagnetism by combining, in quadrature, the effects of changing $\Delta_{E M}$ by one standard deviation with the effects of shifting the average kaon mass squared by $900 \mathrm{MeV}^{2}$, which is the full result of a preliminary computation of electromagnetic effects on this quantity [8]. When computing $f_{D}$, the light valence-quark mass is interpolated to the resulting down-quark mass, and similarly for other quantities involving valence up and down quarks. We expect that the effects of electromagnetic interactions and isospin violation in the sea quarks are small, and ignore them.

Errors from the scale setting and quark mass tuning procedure are estimated from the change in results when $r_{1}$ or $f_{p 4 s}$ are used to set the scale instead of $f_{\pi}$.

\section{Conclusions}

We find preliminary results $f_{D}=209.2(3.0)(3.6) \mathrm{MeV}, f_{D_{s}}=246.4(0.5)(3.6) \mathrm{MeV}, f_{D_{s}} / f_{D}=$ $1.175(16)(11), m_{c} / m_{s}=11.63(4)(9)$ and $m_{u} / m_{d}=0.505(9)(33)$. Our results are consistent with those of other collaborations [10], and the errors are comparable to the most precise published calculation by HPQCD. In the future we will further reduce the error by using staggered chiral 
perturbation theory, which will allow us to make better use of the correlators with unphysical valence-quark masses [7], and by the addition of an ensemble with physical sea-quark masses at a lattice spacing of $0.06 \mathrm{fm}$.

\section{Acknowledgments}

This work was supported by the U.S. Department of Energy and National Science Foundation. Computation for this work was done at the Texas Advanced Computing Center (TACC), the National Center for Supercomputing Resources (NCSA), the National Institute for Computational Sciences (NICS), the National Center for Atmospheric Research (UCAR), the USQCD facilities at Fermilab, and the National Energy Resources Supercomputing Center (NERSC), under grants from the NSF and DOE.

\section{References}

[1] K. Symanzik, In: Recent Developments in Gauge Theories, edited by G. 't Hooft et al. (Plenum Press, New York, 1980), p. 313, (1980); K. Symanzik, Nucl. Phys. B226, 187 (1983); M. Lüscher and P. Weisz, Phys. Lett. B158, 250 (1985); M. Lüscher and P. Weisz, Commun. Math. Phys. 97, 59 (1985); M.G. Alford et al., Phys. Lett. B361, 87 (1995).

[2] E. Follana et al., Nucl. Phys. B (Proc. Suppl.) 129 and 130, 447 (2004) [arXiv:hep-lat/0311004]; E. Follana et al., Nucl. Phys. B. (Proc. Suppl.) 129 and 130, 384, 2004 [arXiv:hep-lat/0406021]; The HPQCD/UKQCD Collaboration: E. Follana et al., Phys. Rev. D75, 054502 (2007) [arXiv:hep-lat/0610092].

[3] A. Bazavov et al. (MILC Collaboration), Phys. Rev. D 82, 074501 (2010), [arXiv:1004.0342].

[4] A. Bazavov et al. (MILC Collaboration), PoS (Lattice 2010) 320 (2010), [arXiv:1012.1265].

[5] A. Bazavov et al. (MILC Collaboration) in preparation.

[6] A. Bazavov et al. (MILC Collaboration), PoS (Lattice2012) 158.

[7] J. Komijani and C. Bernard (MILC Collaboration), PoS (Lattice2012) 199.

[8] L. Levkova et al. (MILC Collaboration), PoS (Lattice2012) 137; C. Bernard et al., PoS (CD12) 030, Proceedings of Chiral Dynamics 2012, in preparation.

[9] C. Aubin et al. (Fermlab lattice and MILC Collaborations), Phys. Rev. Lett. 95, 122002(2005), [arXiv:hep-lat/0506030].

[10] E. Follana et al. (HPQCD Collaboration), Phys. Rev. Lett. 100, 062002 (2008), [arXiv:0706.1726];

C. Davies et al. (HPQCD Collaboration), Phys. Rev. D 82, 114504 (2010), [arxiv:1008.4018];

B. Blossier et al. (ETMC Collaboration), JHEP 0907043 (2009), [arXiv:0904.0954];

Y. Namekawa et al. (PACS-CS Collaboration), Phys. Rev. D 84 (2011) 074505, [arXiv:1104.4600];

P. Dimopoulos et al. (ETMC Collaboration), JHEP 01, 046 (2012), [arXiv:1107.1441];

J.A. Bailey et al. (Fermilab Lattice and MILC collaborations), PoS (Lattice2011) 320

[arXiv:1112.3978]; A. Bazavov et al. (Fermilab Lattice and MILC Collaborations), Phys. Rev. D 85 114506 (2012) [arXiv:1112.3051];

K. Jansen, Trento workshop (2012);

H. Na et al. (HPQCD Collaboration), PoS (Lattice 2012) 102; H. Na et al. (HPQCD

Collaboration), Phys. Rev. D 85125029 (2012) [arXiv:1206.4936]. 